\title{
Sleep Problems and Behavioral Development in Children With Autism Spectrum Disorder: A Literature Review
}

\author{
Bumo Zhang ${ }^{1 *}$ \\ ${ }^{1}$ Graduate School of Education, University of Pennsylvania, Philadelphia, PA 19104, USA \\ "bumoz@gse.upenn.edu
}

\begin{abstract}
.
Sleep problems are common in children with autism spectrum disorder and are associated with problematic behavioral outcomes. The current paper is aimed to provide an overview of the direction of the relationship between sleep problems and behavioral outcomes in children with autism spectrum disorder. Common sleep problems in children with ASD, sleep problems and its related core ASD symptoms, as well as the adverse effects of sleep problems on challenging behavior and adaptive behavior, are well discussed. We argued sleep problems and behavioral problems may be bi-directionally related. Children with ASD are already prone to biological abnormalities, social deficits and repetitive behaviors, which may predispose them to sleep problems. Meanwhile, sleep problems contribute to the development of more intensified ASD symptoms as well as other challenging and maladaptive behavior. We suggested that more longitudinal studies measuring autistic traits and associated sleep problems repeatedly throughout childhood should be conducted to determine if sleep problems and behavioral problems share a common etiology. To treat behavioral problems in children with ASD, clinicians should work with parents to identify sleep problems existed in their children and offer practical guidelines.
\end{abstract}

Key Words: children development, autism spectrum disorder, behavioral problems, sleep problems

\section{Introduction}

Autism spectrum disorder (ASD) is characterized by impairments in social communication and interaction, as well as the presence of restricted and repetitive behaviors [1]. One of 59 children in the United States is estimated to have autism spectrum disorder [2]. Currently, one of the most serious issues reported by parents of children with autism is sleep problems. Children and adolescents with autism spectrum disorders are vulnerable to sleep difficulties. Parent reports indicated that approximately 40$80 \%$ of children with ASD experienced sleep problems in childhood, whereas around $25 \%$ to $40 \%$ of typically developing (TD) children had sleep difficulties [3].

In typically developing children, sleep is associated with multiple aspects of human development, such as memory consolidation and attention [4]. The consequence of disrupted sleep in individuals with ASD is of great concern considering the importance of sleep in daily functioning. Previous research has well documented that sleep problems are associated with core ASD symptoms [5,6,7], as well as other challenging behavior [8,9]. However, the direction of the relationship between sleep problems and behavioral problems in individuals with ASD remains uncertain: Are sleep problems a cause of more behavioral problems in children with ASD? Or are behavioral problems in children with ASD contributed to more sleep problems? Knowing such a relationship will contribute to the literature on autism and provide guidelines for autistic treatment. The current literature review illustrates the relationship between sleep problems and behavioral development in children with ASD through discussing commonly reported sleep difficulties, the potentially adverse effects of sleep problems on core autism symptoms and children's behavior, as well as key limitations in this area.

\section{Common Sleep Problems in ASD}

Children with ASD are significantly more subject to sleep problems than their typically developing cohort $[10,11,12,13]$. In a recent study which recruited ASD children with a mean age of six, sleep problems were nearly two times more common in the ASD group as compared to their age and socioeconomic status matched group of TD children [14]. Two kinds of sleep problems, sleep-onset insomnia, and nocturnal awakenings, have been founded to be the most common issues which children with ASD suffer from.

Sleep-onset insomnia is defined as difficulty falling asleep or staying asleep. Sleep onset and maintenance problems mostly result in reduced total night sleep duration and prolonged periods of awakenings throughout the night [3]. Using the Childhood Sleep Habits Questionnaire (CSHQ), Köse and colleagues found the most frequent sleep problems among the children with ASD were insomnia 
(56.3\%; especially night awakening and decreased sleep duration), bedtime resistance $(73.9 \%)$, and daytime sleepiness (39.6\%) [11]. Difficulty initiating and staying sleep also have implications for settling difficulties, cosleeping, night waking, low sleep efficiency, long sleep latency, early waking, problematic bedtime routines, and daytime sleepiness [15]. Parents of children with ASD reported their children resisted going to bed, had difficulties in initiating sleep, and exhibited nighttime and early morning waking [14].

Another common and consistent sleep problem experienced by children with ASD is nocturnal awakening. Nocturnal awakening refers to the quality of the child's sleep and is often measured by the number and duration of the child's night awakenings [13]. Periods of nocturnal awakening last from 30 minutes up to $2-3 \mathrm{~h}$, where the child may simply laugh or talk, wake in the night, scream, or get up and "play" with toys/objects in the room $[13,16]$. Nocturnal awakenings may result in short sleep duration and daytime sleepiness. The child may also experience prolonged night awakenings, potentially resulting in short sleep duration and/or daytime recovery sleep [15]. Compared to typically developing children, children with ASD often sleep for shorter periods of time during the night and have long periods of awakenings throughout the night [17].

Other less-frequently reported sleep problems in children and adolescents with ASD include parasomnias (e.g., bedwetting, wakes screaming, nightmare disorders), sleeprelated movement disorders (e.g., restless leg, teeth grinding, periodic leg movements, and stereotypic movements), and sleep-related disordered breathing [13]. Using a sample consisted of 112 children in ASD patient group and 112 healthy controls with an age range of 2-18 years, Gunes and colleagues found total score of Children's Sleep Habits Questionnaire (CSHQ), bedtime resistance, and sleep anxiety sub-scores of CSHQ were significantly higher in children with ASD than the control group. Night waking, parasomnias, and sleep-disordered breathing subscores were higher in the patient group, but these findings were not statistically significant [18].

The higher prevalence of sleep problems among children with ASD than in typically developing children may be related to the presence of autism itself. Richdale and Shreck proposed that sleep problems may occur as a result of either i) intrinsic biological or genetic abnormalities that alter brain architecture or biochemistry; ii), psychological or behavioral characteristics connected with core or associated features of ASD; (iii) factors in the family home or environment [3]. Neurohormones like melatonin play a major role in regulating the circadian sleep-wake rhythm [19]. Individuals with ASD had reduced differences between day and night melatonin levels and a relative decreased mean nocturnal melatonin value, compared to the controls [19]. Social difficulties associated with ASD may also play a critical role in the development of sleep problems $[20,21]$. Children with ASD may have difficulties to adequately respond to the social cues related to the timing of sleep behavior, which impact the establishment of a healthy sleep-wake pattern and result in the occurrence of sleep problems. Some studies pointed to environmental factors such as child-rearing practices. Co-sleeping with a parent and family history of sleep problems increased children's risk of having sleep problems [11]. These findings suggested that having an autistic spectrum disorder may increase the risk of having sleep problems. Biological abnormalities that are intrinsic to autism and deficits associated with core ASD features may precipitate children with ASD to sleep problems.

\section{Sleep Problems and Its Related ASD Symptoms}

Sleep problems and autism symptoms may be bidirectionally related. Hollway and Aman argued autism symptom severity and any combination of the autism symptom domains (social deficits and repetitive behaviors) served as vulnerability factors and predisposed children with ASD to sleep problems when presented with environmental stressors [22]. Sleep problems by itself lead to an increase in internalizing and externalizing behaviors which in turn worsen the core autism symptoms. Below, we present the effects of sleep disturbance upon core ASD symptoms - restricted repetitive behavior (RRB) and social impairment, specifically.

\subsection{Repetitive Restricted Behavior}

The repetitive restricted behavior (RRB) compasses a wide range of symptoms, including repetitive language, movements, and use of objects, adherence to nonfunctional routines and rituals, restricted interests, and sensory seeking behaviors, sensitivities, and sensory aversions [1]. $\mathrm{RRB}$ is associated with increased sleep problems, such as bedtime resistance and insomnia [5], as well as short sleep duration [17,23]. Research examining subcategories of repetitive behavior in ASD found repetitive sensory motor (RSM) behavior (repetitive use of objects, complex motor mannerisms, and sensory seeking behaviors) predicted parent-reported sleep problems, even after controlling for anxiety symptoms, whereas insistence on sameness (IS) was not significantly associated with sleep problems [24]. Mazurek and colleagues reported that sensory overresponsibility (SOR) was a longitudinal predictor of sleep problems in young children between 2 to 3 years old. Young children with ASD with high levels of SOR were more likely to experience sleep-onset delay and shortened sleep duration. Sleep disturbance was a longitudinal predictor of the development of hyperactivity and attention problems in very young children with autism. Poor sleepers more likely had self-stimulatory behavior, sensory issues, eating habits hyperactivity, and anxiety [25]. These studies showed that repetitive restricted behavior was a possible predictor of sleep problems. The presence of repetitive restricted behavior increased the likelihood of developing sleep problems, and sleep problems had a negative longitudinal effect on autism symptom. 


\subsection{Social Impairments}

Children with ASD have deficits in social awareness, social information processing, capacity for reciprocal social communication, social anxiety/avoidance, and autistic preoccupations and traits. Thenhausen and colleagues reported a positive association between sleep problems and autism symptom severity, particularly, impairments in social communication, social interaction, and the overall autism index, in adolescents with Asperger syndrome (AS) or high-functioning autism (HFA). Disrupted sleep impaired individuals' competence to react adequately to social key stimuli [26]. Furthermore, the autistic individual's need for social interaction decreased with increased sleep problems. In a sample of 2,714 children with ASD, increased severity of core ASD symptoms was correlated with decreased sleep duration. More severe social impairment was associated with shorter sleep duration when correcting for age and IQ [17]. Consistent with their finding, Phung and Goldberg found more sleepwake problems and more daytime sleepiness were associated with more discord with peers in adolescents with ASD instead of the TD group [7]. Increased sensitivity to environmental stimuli in the bedroom and screaming at night also predicted communication problems [16]. These findings may be inferred that in a child with ASD, who is already prone to social impairment, shorter sleep duration that may further intensify autistic symptomatology during the day.

\section{Sleep Problems and Its Effects on Behavioral Development}

Sleep problems in autism are associated with multiple aspects of behavioral development. A bunch of studies has found that sleep problems, such as shorter sleep duration and frequent awakenings at night, predict challenging behaviors and maladaptive functioning. Children with ASD and sleep problems are at higher risk of displaying challenging behavior and having poor adaptive functioning, compared to children with ASD without sleep problems [16,17]. In this case, sleep problems may exacerbate behavioral problems among children with ASD. In the following paragraph, we discuss the possible adverse effects of sleep problems on challenging behavior and adaptive behavior.

\subsection{Challenging Behavior}

Challenging behaviors are common in children with ASD, which usually occur during the toddler years [27]. Previous literature has defined challenging behavior as intense and persistent 'culturally abnormal' behaviors which are disruptive and destructive in nature [19,24]. Children with ASD and sleep problems exhibit more daytime problematic behavior than children with ASD and no sleep problems $[5,9]$. Challenging behaviors include internalizing behaviors and externalizing behaviors. Below, we further discuss the association between sleep problems and these two behavioral problems.

\subsubsection{Internalizing Behavior.}

Internalizing behavior, namely, means behavior that is lashing out at the self. Children with ASD and sleep problems are at great risk of exhibiting internalizing behaviors, such as affective problems (e.g., anxiety, depression, anger), self-injurious behavior (any actions that cause or have the potential to cause physical harm or injury to self), social withdrawal problems, inattention [14], and stereotypes (repetitive movements and behavior) [23,24]. Among 193 preschool-aged Japanese children with ASD, the severity of sleep problems, especially insomnia, was significantly correlated with behavioral problems in ASD preschoolers [8]. Using the Child Behavior Checklist, Park and colleagues found autistic children with sleep problems were more likely to have withdrawal problems, somatizing problems, and total internalizing behavior problems (i.e., anxious, depressive, and over-controlled), compared to those without sleep problems [5]. In a study using the Parental Concerns Questionnaire (PCQ) to characterize children with ASD as good and bad sleepers, poor sleepers had the highest overall risk of exhibiting self-injurious behaviors even after the model was adjusted for age. Almost a fourth of poor sleepers were reported to have selfinjurious behavior. The relative risk of being a poor sleeper rather than a good sleeper if the child had such behavioral problems was $10 \%$ [28]. These researches highlight the strong association between sleep problems and internalizing challenging behavior, and more importantly, provide evidence which supports the assumption that sleep problems may worsen internalizing behavior problems.

\subsubsection{Externalizing Behavior.}

Externalizing behavior refers to behavior that is directed toward the external environment. Common aspects of externalizing behaviors investigated within the literature in ASD include hyperactivity (behaviors such as fidgeting and running about or climbing excessively), non-compliance, oppositional behavior, tantrums, property destruction, and aggression [29].

A substantial body of research has focused on the relationship between sleep problems and externalizing problematic behavior. ASD children with sleep problems are more likely to exhibit externalizing behavior problems, especially aggressive behaviors, noncompliant behavior, and hyperactivity $[8,24,28,29]$. Some research suggested that sleep problems may intensify behavioral problems already existed in children with ASD [16]. Increased severity of sleep problems was found to be associated with increased ratings of challenging behavior for total and externalizing behavior problems in children and adolescents with ASD. People in this population who 
suffered from more severe sleep disturbance engaged in more externalizing behavior problems than those with relatively mild sleep problems [24]. Therefore, it is reasonable to propose that sleep problems may exacerbate externalizing behavior problems.

\subsection{Adaptive Behavior}

Adaptive behavior is defined as one's ability to be selfsufficient in real life situations [30]. A huge body of research showed that children with ASD exhibited significant deficits in their adaptive skills [30]. Adaptive behavior of children with ASD, as mostly measured by the Vineland Adaptive Behavior Scales (VABS), consists of four domains: Communication, Daily Living Skills (DLS), Socialization, and Motor Skills [31].

Sleep duration is significantly associated with children's adaptive skills. Taylor, Schreck, and Mulick found children who slept fewer hours per night had lower overall intelligence, verbal skills, overall adaptive functioning, daily living skills, socialization skills, and motor development [32]. For the Motor composite, a study comparing seventy-three children with ASD and their age and sex matched TD group, found poor motor skills of VABS were significantly associated with sleep problems as well as "more than others" response to movement processing on sensory profile [33]. For the communication domain, children who slept fewer hours at night with waking during the night had more communication problems [32]. Additionally, Schreck and colleagues found increased sensitivity to environmental stimuli in the bedroom and screaming at night predicted communication problems [16]. For the DLS domain, children who slept less on average per night displayed more deficits in skills needed to complete typical daily living tasks such as hygiene, eating, and toileting [32]. For the socialization domain, fewer hours of sleep per night predicted overall autism scores and social skills deficits [16,17]. These results suggested that sleep problems negatively impacted children's adaptive functioning.

A study investigating the links between adaptive skills and the severity of sleep problems showed robust results. In a sample of children with ASD between the ages of 4 to 10 , Sikora and colleagues found only the motor composite and DLS scores differed between the mild and moderate to severe sleep groups [9]. Specifically, children with moderate to severe sleep problems had the lowest composite score than children with mild to moderate sleep problems. The good sleep group scored significantly lower on the Communication domain than both the mild and moderate to severe sleep problem groups for the preschoolaged children but only significantly lower on the Communication domain than the moderate to severe sleep problem group for the school-aged children. For the DLS, there were no differences in scores between the good sleep and mild sleep problem groups for both preschool- and school-aged children, but there were differences in scores between the good sleep group and the moderate to severe sleep group for preschool-aged children. For the socialization domain, there were no differences in scores across groups for the preschool-aged children, but there were differences in scores between the good sleep group and the sleep problem groups for children 6 to 10 years old. There were no differences between the mild and moderate to severe sleep problem groups for any domain at any age. One possible explanation for these results is differences in VABS-II domains. The Communication and Socialization domains, in general, reflect core ASD symptoms, whereas the DLS and motor domain reflect specific skill sets [9]. These findings may be interpreted as in a child with ASD, sleep problems may intensify autistic symptomatology which further negative impact their daily functioning.

\section{The Direction of the Relationship Between Behavioral Problems and Sleep Problems}

Studies on sleep problems in ASD have touched on a common question: Do sleep problems cause or exacerbate behavioral problems in children with ASD? Or do behavioral problems cause or exacerbate sleep problems in this population? Some studies proposed that sleep problems may exacerbate problems already present in children with ASD. Schreck and colleagues argued that sleep problems may be a possible predictor of intensified symptoms of autism. They found shorter sleep duration at night predicted overall autism scores and social skills deficits. Similarly, fewer hours of sleep per night and screaming during the night predicted stereotypic behavior. Increased sensitivity to environmental stimuli in the bedroom and screaming at night predicted communication problems [16]. In addition to exerting negative influences on core ASD symptomatology, sleep problems also appear to worsen challenging behavior and adaptive functioning which are commonly exhibited by children in this population. Adams and colleagues found a main effect of severity of sleep problems for total challenge behavior and externalizing challenging behavior [24]. Similarly, Malhi and colleges found total scores on Children's Sleep Habits Questionnaire (CSHQ) and duration of night awake time explained 30\% of the variance in the number of daytime behavioral problems in their multiple regression analysis [14]. These studies fairly indicate that sleep problems may intensify behavioral problems in children with ASD.

In contrast to this line of thinking, some research provides support for the opposite direction: behavioral problems appear to predict sleep problems in children with ASD. In a sample of psychiatrically hospitalized children with ASD, patients who displayed more maladaptive behaviors at admission, were found to have a shorter sleep duration when they were nearing discharge [34]. This study demonstrated a significant correlation between behavioral problems at baseline and future sleep duration. Similarly, a longitudinal study found aggressive behavior at baseline predicted poor sleep at the first annual follow-up [35]. In this case, sleep problems may be exacerbated by behavioral problems. 
Instead of suggesting a unidirectional causality between sleep problems and behavioral problems among children with ASD, we argue that it is more likely to be a bidirectional relationship: behavioral problems that children with ASD are already prone to (social deficits and repetitive behavior) may predispose children with ASD to sleep problems; meanwhile, poorer sleep may contribute to the development of more intensified ASD symptoms as well as other challenging and maladaptive behavior. Our assumption is supported by findings from a longitudinal study. Mazurek and his colleagues found that sleep problems at baseline predicted later development of ADHD symptoms in younger children and somatic complaints in older children. For younger children, sensory overresponsivity predicted future sleep problems. Their finding suggested that sensory over-reactivity may contribute to sleep problems in young children with ASD, which may further result in poor daytime functioning [25]. Several studies have found a positive correlation between sleep problems and the severity of autistic symptoms. Mayes and Calhoun noticed parent ratings of autism severity, hyperactivity, mood variability, and aggression were the strongest predictors of sleep disturbance [36]. Consistent with their work, Tudor and colleagues also found sleep onset delay and sleep duration were positively correlated with autism symptoms and autism severity. More importantly, they found sleep onset delay was the strongest predictor of communication deficit, stereotyped behavior, and autism severity [37]. These results provide support for a bidirectional relationship between sleep problems and behavioral problems.

If such direction is true, interventions addressing behavioral problems in children with ASD may include solutions for sleep problems in their work. Clinicians should be aware that the development of problematic behavior may be contributed to the presence of sleep problems. Therefore, in addition to providing suggestions for treating behavioral problems, clinicians should also work closely with parents to identify sleep problems existed in their children and offer practical guidelines.

\section{Limitation and Future Direction}

A major limitation existed in the current field is a lack of clear definitions of clinically relevant sleep problems in pediatric populations. Researchers typically construct their own criteria or definitions of sleep problems, such as cutoff or frequency scores on sleep questionnaires or diaries [3], which result in various forms of research questions on sleep problems in ASD. The issue of sleep problem instrument is further complicated when measuring children's sleep. Most studies used parent report to assess children's sleep. However, the measurement and description of childhood sleep problems, especially for children with ASD, may differ as a parent or another responsible adult (e.g., teacher) generally reports and describes the problem. It may be challenging for parents to accurately report on internally experienced symptoms, such as anxiety or somatic symptoms $[3,25]$. Therefore, future studies should develop more appropriate and specific definitions of sleep problems for children with ASD. Clear definitions of sleep problems in ASD will provide a more accurate examination of trajectories of sleep problems.

Secondly, the majority of studies on sleep in children with ASD used the cross-sectional design and include clinical samples [14]; and the few longitudinal studies lacked baseline measures and diagnosis of ASD [16,21,38]. Most studies compared children with autism to the typical developing cohorts. In this case, the impact of confounding factors like marital discord, poor maternal health, and poor parenting, may not be eliminated [5]. To further explore the relationship between sleep problems and ASD, more longitudinal studies should measure autistic traits and associated sleep problems repeatedly throughout childhood. Cross-sectional studies should also recruit comparison groups like twins and resident siblings.

Additionally, more research should be conducted to determine if sleep problems and behavioral problems share a common etiology. While most research supported a bidirectional relationship between sleep problems and behavioral problems in children with ASD [25], Verhoeff and colleagues argued that sleep problems did not precede and worsen autistic behavior but rather co-occur with autistic traits in early childhood. They found sleep problems in toddlerhood were associated with autistic traits in middle childhood, but this association was not significant when adjusting for early autistic traits [21]. In contrast, autistic traits and a diagnosis of ASD in childhood were associated with sleep problems at later ages. One possible explanation for differences is that this study used the Child Behavior Checklist, an instrument which was primarily used to assess behavioral problems in children with ASD, to measure sleep problems. Thus, differences in sleep measurement may have influenced outcomes. These inconsistent findings have called for more rigorous research which measures autistic traits and associated sleep problems repeatedly throughout childhood.

Thirdly, treatments of autism may benefit from adopting different strategies. Future research should consider the relative effectiveness of various treatments (e.g., medication, behavioral treatment, or cognitive- behavioral therapy) for different types of sleep disturbance across childhood.

\section{Conclusion}

This paper provides a review of the relationship between sleep problems and behavioral development in children with autism spectrum disorders. In summary, we argue sleep problems and behavioral problems may be bidirectionally related. Children with ASD are already prone to biological abnormalities, social deficits and repetitive behaviors, which may predispose them to sleep problems. Meanwhile, sleep problems contribute to the development of more intensified ASD symptoms as well as other challenging and maladaptive behavior. Prospective studies should adopt a multi-method and interdisciplinary approach to understand the links between sleep problems 
and behavioral outcomes in children with ASD. The treatment of behavioral problems in children with ASD may also include solutions for sleep problems in their work.

\section{References}

[1] American Psychiatric Association. (2013). Diagnostic and statistical manual of mental disorders (5th ed.). Arlington, VA: American Psychiatric Association.

[2] Baio, J., Wiggins, L., Christensen, D. L., Maenner, M. J., Daniels, J., Warren, Z., . . . Dowling, N. F. (2018). Prevalence of autism spectrum disorder among children aged 8 years - autism and developmental disabilities monitoring network, 11 sites, united states, 2014. Morbidity and Mortality Weekly Report. Surveillance Summaries (Washington: 2002), 67(6), 123.

[3] Richdale, A. L., \& Schreck, K. A. (2009). Sleep problems in autism spectrum disorders: Prevalence, nature, \& possible biopsychosocial aetiologies. Sleep Medicine Reviews, 13(6), 403-411.

[4] Ashworth, A., Hill, C. M., Karmiloff-Smith, A., \& Dimitriou, D. (2014). The Importance of Sleep: Attentional Problems in School-Aged Children With Down Syndrome and Williams Syndrome. Behavioral Sleep Medicine, 13(6), 455-471.

[5] Park, S., Park, T., Cho, I. H., Cho, S., Kim, B., Kim, J., . . . Yoo, H. J. (2012). Sleep problems and their correlates and comorbid psychopathology of children with autism spectrum disorders. Research in Autism Spectrum Disorders, 6(3), 1068-1072.

[6] Hundley, R. J., Shui, A., \& Malow, B. A. (2016). Relationship between subtypes of restricted and repetitive behaviors and sleep disturbance in autism spectrum disorder. Journal of Autism and Developmental Disorders, 46(11), 3448-3457.

[7] Phung, J. N., \& Goldberg, W. A. (2017). Poor sleep quality is associated with discordant peer relationships among adolescents with autism spectrum disorder. Research in Autism Spectrum Disorders, 34(Complete), 10-18.

[8] Hirata, I., Mohri, I., Kato-Nishimura, K., Tachibana, M., Kuwada, A., Kagitani-Shimono, K., . . . Taniike, M. (2016). Sleep problems are more frequent and associated with problematic behaviors in preschoolers with autism spectrum disorder. Research in Developmental Disabilities, 49-50, 86-99.
[9] Sikora, D. M., Johnson, K., Clemons, T., \& Katz, T. (2012). The relationship between sleep problems and daytime behavior in children of different ages with autism spectrum disorders. Pediatrics, 130(Supplement 2), S83-S90.

[10] Gail Williams, P., Sears, L. L., \& Allard, A. (2004). Sleep problems in children with autism. Journal of Sleep Research, 13(3), 265-268.

[11] Köse, S., Yılmaz, H., Ocakoğlu, F. T., \& Özbaran, N. B. (2017). Sleep problems in children with autism spectrum disorder and intellectual disability without autism spectrum disorder. Sleep Medicine, 40, 69-77.

[12] Mutluer, T., Karakoc Demirkaya, S., \& Abali, O. (2016). Assessment of sleep problems and related risk factors observed in turkish children with autism spectrum disorders. Autism Research, 9(5), 536-542.

[13] Reynolds, A. M., Soke, G. N., Sabourin, K. R., Hepburn, S., Katz, T., Wiggins, L. D., . . . Levy, S. E. (2019). Sleep problems in 2- to 5-year-olds with autism spectrum disorder and other developmental delays. Pediatrics, 143(3), e20180492.

[14] Malhi, P., Kaur, A., Singhi, P., \& Sankhyan, N. (2018). Sleep dysfunction and behavioral daytime problems in children with autism spectrum disorders: A comparative study. The Indian Journal of Pediatrics, 86(1), 12-17.

[15] Abel, E., Kim, S. Y., Kellerman, A. M., \& Brodhead, M. T. (2017). Recommendations for identifying sleep problems and treatment resources for children with autism spectrum disorder. Behavior Analysis in Practice, 10(3), 261-269.

[16] Schreck, K. A., Mulick, J. A., \& Smith, A. F. (2004). Sleep problems as possible predictors of intensified symptoms of autism. Research in Developmental Disabilities, 25(1), 57-66.

[17] Veatch, O. J., Sutcliffe, J. S., Warren, Z. E., Keenan, B. T., Potter, M. H., \& Malow, B. A. (2017). Shorter sleep duration is associated with social impairment and comorbidities in ASD. Autism Research, 10(7), 1221-1238.

[18] Gunes, S., Ekinci, O., Feyzioglu, A., Ekinci, N., \& Kalinli, M. (2019). Sleep problems in children with autism spectrum disorder: Clinical correlates and the impact of attention deficit hyperactivity disorder. Neuropsychiatric Disease and Treatment, 15, 763-771. 
[19] Matson, J. L., Neal, D., Fodstad, J. C., \& Hess, J. A. (2010). The relation of social behaviors and challenging behaviors in infants and toddlers with autism spectrum disorders.Developmental Neurorehabilitation, 13(3), 164-169.

[20] May, T., Cornish, K., Conduit, R., Rajaratnam, S. M. W., \& Rinehart, N. J. (2015). Sleep in highfunctioning children with autism: Longitudinal developmental change and associations with behavior problems. Behavioral Sleep Medicine, 13(1), 2-18.

[21] Verhoeff, M. E., Blanken, L. M. E., Kocevska, D., Mileva-Seitz, V. R., Jaddoe, V. W. V., White, T., . . Tiemeier, H. (2018). The bidirectional association between sleep problems and autism spectrum disorder: A population-based cohort study. Molecular Autism, 9(1), 8-9.

[22] Hollway, J. A., \& Aman, M. G. (2011). Sleep correlates of pervasive developmental disorders: A review of the literature. Research in Developmental Disabilities, 32(5), 1399-1421.

[23] Abel, E. A., Schwichtenberg, A. J., Brodhead, M. T., \& Christ, S. L. (2018). Sleep and challenging behaviors in the context of intensive behavioral intervention for children with autism. Journal of Autism and Developmental Disorders, 48(11), 3871-3884.

[24] Adams, H. L., Matson, J. L., \& Jang, J. (2014). The relationship between sleep problems and challenging behavior among children and adolescents with autism spectrum disorder. Research in Autism Spectrum Disorders, 8(9), 1024-1030.

[25] Mazurek, M. O., Dovgan, K., Neumeyer, A. M., \& Malow, B. A. (2019). Course and predictors of sleep and co-occurring problems in children with autism spectrum disorder. Journal of Autism and Developmental Disorders, 49(5), 2101-2115.

[26] Thenhausen, N., Kuss, M., Wiater, A., \& Schlarb, A. A. (2017). Sleep problems in adolescents with asperger syndrome or high-functioning autism. Somnologie, 21(S1), 28-36.

[27] Murphy, G. H., Beadle-Brown, J., Wing, L., Gould, J., Shah, A., \& Holmes, N. (2005). Chronicity of challenging behaviors in people with severe intellectual disabilities and/or autism: A total population sample. Journal of Autism and Developmental Disorders, 35(4), 405-418.

[28] Goldman, S. E., McGrew, S., Johnson, K. P., Richdale, A. L., Clemons, T., \& Malow, B. A. (2011).
Sleep is associated with problem behaviors in children and adolescents with autism spectrum disorders. Research in Autism Spectrum Disorders, 5(3), 1223-1229.

[29] Mazurek, M. O., \& Sohl, K. (2016). Sleep and behavioral problems in children with autism spectrum disorder. Journal of Autism and Developmental Disorders, 46(6), 1906-1915.

[30] Kanne, S. M. , Gerber, A. J. , Quirmbach, L. M. , Sparrow, S. S. , Cicchetti, D. V. , \& Saulnier, C. A. . (2011). The role of adaptive behavior in autism spectrum disorders: implications for functional outcome. Journal of Autism and Developmental Disorders, 41(8), 1007-1018.

[31] Sparrow, S. S., Balla, D. A., \& Cicchetti, D. V. (1984). The Vineland Adaptive Behavior Scales: Interview edition, expanded form. Circle Pines, MN: American Guidance Service.

[32] Taylor, M. A., Schreck, K. A., \& Mulick, J. A. (2012). Sleep disruption as a correlate to cognitive and adaptive behavior problems in autism spectrum disorders. Research in Developmental Disabilities, 33(5), 1408-1417.

[33] Tyagi, V., Juneja, M., \& Jain, R. (2019). Sleep problems and their correlates in children with autism spectrum disorder: An indian study. Journal of Autism and Developmental Disorders, 49(3), 1169-1181.

[34] Sannar, E. M., Palka, T., Beresford, C., Peura, C., Kaplan, D., Verdi, M., . . . For the Autism and Developmental Disorders Inpatient Research Collaborative (ADDIRC). (2018). Sleep problems and their relationship to maladaptive behavior severity in psychiatrically hospitalized children with autism spectrum disorder (ASD). Journal of Autism and Developmental Disorders, 48(11), 3720-3726.

[35] Shui, A. M., Katz, T., Malow, B. A., \& Mazurek, M. O. (2018). Predicting sleep problems in children with autism spectrum disorders. Research in Developmental Disabilities, 83, 270-279.

[36] Mayes, S. D., \& Calhoun, S. L. (2009). Variables related to sleep problems in children with autism. Research in Autism Spectrum Disorders, 3(4), 931-941.

[37] Tudor, M. E., Hoffman, C. D., \& Sweeney, D. P. (2012). Children with autism: sleep problems and symptom severity. Focus on Autism and Other Developmental Disabilities, 27(4), 254-262. 
[38] Sivertsen, B., Posserud, M., Gillberg, C., Lundervold, A. J., \& Hysing, M. (2012). Sleep problems in children with autism spectrum problems: A longitudinal population-based study. Autism: The International Journal of Research and Practice, 16(2), 139 